\title{
Pilot study to assess the safety of local lidocaine injections during endoscopic submucosal dissection for early gastric cancer
}

\author{
Shinsuke Kiriyama ${ }^{1,2}$, Ichiro Oda ${ }^{1}$, Fumiya Nishimoto ${ }^{1}$, Yumi Mashimo $^{1}$, Hisatomo Ikehara $^{1}$, \\ and TAKUJI GOTODA ${ }^{1}$ \\ ${ }^{1}$ Endoscopy Division, National Cancer Center Hospital, 5-1-1 Tsukiji, Chuo-ku, Tokyo 104-0045, Japan \\ ${ }^{2}$ Department of General Surgical Science, Graduate School of Medicine, Gunma University, Maebashi, Japan
}

\begin{abstract}
Background. In Japan, endoscopic submucosal dissection (ESD) for early gastric cancer (EGC) is performed by endoscopists on patients under sedation. There is an increased risk of anesthesia-related complications due to the higher sedative doses required during lengthier ESDs, so we sought to determine whether a local pain control method could safely reduce such doses.

Methods. Twenty EGC patients enrolled in this study received local lidocaine injections during ESDs at our hospital (lidocaine group; LG). Electrocardiography, heart rate, oxygen saturation, and blood pressure were monitored during and after the ESDs, along with the doses of midazolam and pentazocine. Pain assessments were recorded for LG patients on the day of their ESDs and the following day.

Results. The mean volume of lidocaine injection solution was $55.4 \mathrm{ml}$ and the mean dose of lidocaine was $236 \mathrm{mg}$ (range, 100-300 mg). The mean size of the resected specimens was $39.3 \mathrm{~mm}$ and mean procedure time was $66.0 \mathrm{~min}$. There were no lidocaine-related complications, and electrocardiography, heart rate, oxygen saturation and blood pressure measurements were normal. In comparison to 157 consecutive patients (control group; CG), who had similar characteristics and had undergone ESDs previously with submucosal injections of conventional normal saline solution, the mean \pm SD pentazocine dose of $15.8 \pm 10.3 \mathrm{mg}$ in the LG was significantly lower $(P<0.01)$ than the dose of $23.1 \pm 9.5 \mathrm{mg}$ in the $\mathrm{CG}$, and none of the LG patients complained of abdominal pain on the day of their ESDs, whereas such pain was reported by $17 \%$ (27/157) of the CG.

Conclusion. Local lidocaine injections into the submucosal layer were safe when administered during ESDs performed on EGC patients under sedation.
\end{abstract}

Key words Lidocaine - Endoscopic submucosal dissection $($ ESD) $\cdot$ Early gastric cancer $\cdot$ Local anesthesia $\cdot$ Submucosal injection

Offprint requests to: S. Kiriyama

Received: November 4, 2008 / Accepted: May 28, 2009

\section{Introduction}

Although endoscopic submucosal dissection (ESD) is less invasive for early gastric cancer (EGC) than gastrectomy, the classical open surgical procedure, some ESD patients suffer considerable postoperative pain. Localized pain both during and after ESD for large EGCs is probably caused by ulcer defects and/or electrical thermal burns extending from the submucosal (sm) layer to the serosa.

There is a problem not only with pain management but also with sedation management during the ESD procedure. In a previous report, $41 \%$ of ESDs took longer than $1 \mathrm{~h} \mathrm{[1]}$. As a result of such increased procedure times, therefore, higher doses of sedative drugs such as midazolam have become necessary, and endoscopists performing ESDs must be even more careful about the depth of patient sedation, because deep sedation can occur during endoscopic procedures [2]. Because there is an increased risk of anesthesia-related complications with the higher sedative drug doses required during lengthier ESDs, we decided to determine whether a local pain control method could safely reduce such doses.

By controlling localized pain during ESD, we thought that it might be possible to reduce the dose of the sedative drug administered during the actual procedure. In addition, administering local analgesia for pain during ESD would amount to providing preemptive analgesia for patient pain that might otherwise be experienced the day after the procedure [3].

Local anesthesia is commonly used in surgery, including laparoscopic surgery $[4,5]$, but there have been no previous reports on the use of lidocaine for local pain control either during or after ESD. This study intended to assess the safety of local lidocaine injections into the sm layer of EGC lesions in controlling localized pain both during and subsequent to ESD procedures performed on patients under sedation. 


\section{Patients and methods}

\section{Patients}

A total of 20 EGC patients were enrolled in this study (lidocaine group; LG) between September 2005 and April 2007 at the National Cancer Center Hospital in Tokyo, Japan. These subjects were scheduled for gastric ESDs based on endoscopic mucosal resection (EMR) guideline criteria $[6,7]$ and had their given informed consent before undergoing the procedures. Patients with: (1) an allergy to lidocaine or other amide-type local anesthesia; (2) severe liver disease, heart disease, or renal dysfunction; (3) a gastric or duodenal ulcer; (4) atherosclerotic disease; (5) hyperthyroidism; or (6) an American Society of Anesthesiologists (ASA) physical status higher than class 2 were excluded from the study. This study was performed in accordance with the Helsinki Declaration as revised in 1989.

\section{ESD procedures}

ESDs were performed following a standard protocol, and procedure times were recorded for all LG patients. Lesion margins were delineated beforehand by using $0.2 \%$ indigo-carmine dye spraying. After the marking dots were made with a needle knife (KD-1L-1; Olympus Medical Systems, Tokyo, Japan), 0.5\% lidocaine solution was injected into the sm layer to lift the lesion. A circumferential incision in the mucosa was then completed using the needle knife, an insulation-tipped knife (IT knife; KD-610L, Olympus) [7-10], and a highfrequency current electrical generator (ICC 200; ERBE, Tubingen, Germany) and, finally, the thickened sm layer was dissected using the same two endoscopic knives.

\section{Injection solutions}

As previously noted, we used $0.5 \%$ lidocaine solution consisting of $10 \mathrm{ml}$ of Xylocaine Polyamp 1\%, containing $100 \mathrm{mg}$ of lidocaine hydrochloride $(\mathrm{HCl}$; AstraZeneca, Osaka, Japan), $9.7 \mathrm{ml}$ of normal saline, $0.2 \mathrm{ml}$ of $0.4 \%$ indigo-carmine dye, and $0.1 \mathrm{ml}$ of $0.1 \%$ epinephrine (Table 1) for the LG in this study. The maximum volume of $0.5 \%$ lidocaine solution per LG patient was $60 \mathrm{ml}$, which contained $300 \mathrm{mg}$ of lidocaine $\mathrm{HCl}$ [11-13].

For the purpose of lifting an EGC lesion, the lidocaine injection solution was injected into the sm layer under and around the lesion, as determined by the endoscopist, using a 23-gauge needle injection catheter (NM-200L-0423; Olympus). Once the needle was inserted into the sm layer, the endoscopist's assistant would aspirate the syringe, particularly for those patients suffering from hypertension or diabetes mellitus, thereby creating negative pressure. If no blood reflex appeared, the assistant would begin injecting the solution and would stop when the lesion was sufficiently lifted and became slightly blue in color, with the total volume of the injection solution being recorded at that time.

\section{Patient monitoring during and following ESD procedures}

Electrocardiography, heart rate, oxygen saturation, and blood pressure were automatically monitored during and after each ESD; lidocaine-related complications were recorded, as were any complications resulting from the ESD itself, such as a perforation. In addition, the patient's white blood cell count and C-reactive protein level were checked on the first day following the ESD.

\section{Sedation during ESDs}

All ESDs on LG patients were performed following standard sedation procedures. Sedation was induced with $0.06 \mathrm{mg} / \mathrm{kg}$ of midazolam. Incremental 2-mg doses of midazolam were given if a patient demonstrated signs of discomfort, restlessness, or agitation, or responded to verbal commands. When additional midazolam was ineffective, $15 \mathrm{mg}$ of pentazocine was infused as an analgesic agent. Each patient received oxygen by nasal cannula from the start of the ESD for a maximum period of $3 \mathrm{~h}$ after the procedure.

Table 1. Composition of injection solutions

\begin{tabular}{lcc}
\hline & Lidocaine solution & Conventional solution \\
\hline $\begin{array}{l}\text { 1\% Lidocaine }(\mathrm{ml}) \\
\quad \text { Lidocaine } 100 \mathrm{mg} / 10 \mathrm{ml})\end{array}$ & 10 & 0 \\
Normal saline $(\mathrm{ml})$ & 9.7 & 19.7 \\
$0.4 \%$ Indigo-Carmine $(\mathrm{ml})$ & 0.2 & 0.2 \\
$0.1 \%$ Epinephrine $(\mathrm{ml})$ & 0.1 & 0.1 \\
Lidocaine concentration $(\%)$ & 0.5 & 0 \\
Maximum lidocaine injection & $300 \mathrm{mg} / 60 \mathrm{ml}$ & \\
\hline
\end{tabular}


Table 2. Results

\begin{tabular}{lc}
\hline & Lidocaine group (LG) \\
\hline Number & 20 \\
Sex & 16 \\
Male & 4 \\
Female & 55.4 \\
Injection solution & 236 \\
Mean injection volume (ml) & $(100-300)$ \\
Lidocaine, mg & \\
(range) & $8700 \pm 2100$ \\
Laboratory data (day 1) & $0.8 \pm 0.7$ \\
Mean +/- SD white blood cell count & \\
C-Reactive protein (g/dl) & 0 \\
Abnormality in monitoring & 0 \\
Electrocardiography & 0 \\
Heart rate & 0 \\
Oxygen saturation & \\
Blood pressure & \\
Complications & \\
Lidocaine-related & \\
$\quad$ Intoxication & 0 \\
$\quad$ Convulsion & 0 \\
$\quad$ Arrhythmia & 0 \\
Respiratory & \\
Perforation & 0 \\
\hline
\end{tabular}

In assessing the necessity for sedative drugs, we retrospectively compared the doses of midazolam and pentazocine during ESDs performed on the $20 \mathrm{LG}$ patients with the doses used in 157 other consecutive EGC patients who had previously received conventional sm injections during ESDs, as a historical control group (control group; CG). The ESDs in the CG patients, whose clinical characteristics matched those of the LG patients, had been performed between January and August 2005, with the patients under sedation, as these CG patients also met the EMR guideline criteria. Their conventional sm injections consisted of a solution of $19.7 \mathrm{ml}$ of normal saline, $0.2 \mathrm{ml}$ of $0.4 \%$ indigo-carmine dye, and $0.1 \mathrm{ml}$ of $0.1 \%$ epinephrine (Table 1).

\section{Evaluation of abdominal pain after ESD}

In evaluating the efficacy of pain control, a written questionnaire about the absence or presence of abdominal pain (no pain, mild pain without painkiller, or severe pain with painkiller) was distributed to each patient in the LG, to be completed on the day of the ESD after the procedure, and on the next day. We then proceeded to retrospectively determine the absence or presence of abdominal pain for each CG patient at the same two points in time as those in the LG, based on complete medical records. Finally we also identified those patients in each group who either received a painkiller (pain [+]) or did not receive a painkiller (pain [-]) after their procedures.

\section{Statistical analysis}

Values for all variables in this study are expressed as means \pm SD. In comparing baseline characteristics between the two groups, we used a $t$-test for continuous variables, with the $\chi^{2}$ or Fisher test for dichotomous variables. All statistical analyses were performed using the Statistical Package for the Social Sciences (SPSS) program (SPSS, version 8.0 for Windows; Tokyo, Japan). The $P$ values were two-sided, and $P<0.05$ determined statistical significance.

\section{Results}

The mean volume of lidocaine injection solution was $55.4 \mathrm{ml}$ and the mean dose of lidocaine was $236 \mathrm{mg}$ (range, 100-300 mg; Table 2). There were no electrocardiography, heart rate, oxygen saturation, or blood pressure abnormalities recorded, nor were there any episodes of lidocaine intoxication, including respiratory depression or hypotension, convulsion, or arrhythmia, such as cardiovascular collapse or bradycardia. The mean \pm SD white blood cell counts and C-reactive protein values on the first post-procedure day were $8700 \pm 2100$ cells $/ \mathrm{mm}^{3}$ and $0.8 \pm 0.7 \mathrm{~g} / \mathrm{dl}$, respectively.

\section{Pain evaluation in comparison with historical control group}

In our comparison of LG patients with the CG as a historical control, there were no significant differences 
Table 3. Comparison with historical control

\begin{tabular}{lccc}
\hline & Lidocaine group & Control group & $P$ value \\
\hline Number & 20 & 157 & \\
Age, years (mean \pm SD) & $69.4 \pm 7.2$ & $66.7 \pm 9.4$ & $\mathrm{NS}$ \\
Resection size, mm (mean \pm SD) & $39.3 \pm 11.6$ & $36.3 \pm 8.3$ & $\mathrm{NS}$ \\
Sedating agents & & & \\
$\quad$ Midazolam, mg (mean \pm SD) & $9.7 \pm 3.2$ & $10.3 \pm 4.6$ & $\mathrm{NS}$ \\
$\quad$ Pentazocine, mg & $15.8 \pm 10.3$ & $23.1 \pm 9.5$ & $<0.01$ \\
Procedure time (min) & $66.0 \pm 36.9$ & $61.0 \pm 30.7$ & $\mathrm{NS}$ \\
Post-ESD pain & & & \\
Day 0 & $20(100 \%)$ & $130(83 \%)$ & \\
$\quad$ Pain (-) & 0 & $27(17 \%)$ & $<0.05$ \\
$\quad$ Pain (+) & $18(90 \%)$ & $95(61 \%)$ & \\
Day 1 & $2(10 \%)$ & $62(39 \%)$ & $<0.05$ \\
$\quad$ Pain (-) & & & \\
$\quad$ Pain (+) & &
\end{tabular}

NS, not significant

in clinicopathological characteristics between the two groups. The mean $\pm \mathrm{SD}$ size of the resected specimens was $39.3 \pm 11.6 \mathrm{~mm}$ in the $\mathrm{LG}$ and $36.3 \pm 8.3 \mathrm{~mm}$ in the $\mathrm{CG}$, while the mean $\pm \mathrm{SD}$ ages were $69.4 \pm 7.2$ years and $66.7 \pm 9.4$ years, respectively (Table 3 ). The mean \pm SD doses of midazolam were $9.7 \pm 3.2 \mathrm{mg}$ and $10.3 \pm 4.6 \mathrm{mg}$ in the LG and CG, respectively (difference not significant [NS]), but the mean \pm SD dose of pentazocine in the LG was significantly lower than that in the CG, at $15.8 \pm 10.3 \mathrm{mg}$ and $23.1 \pm 9.5 \mathrm{mg}$, respectively $(P<$ $0.01)$.

All of the LG patients completed the questionnaires regarding the absence or presence of abdominal pain on the day of the ESD following the procedure, as well as the next day. None of the LG patients complained of abdominal pain immediately following their ESDs, whereas abdominal pain that required a painkiller occurred in $17 \%(27 / 157)$ of the CG patients $(P<0.05)$. On the day after their ESDs, $2(10 \%)$ of the LG patients complained of abdominal pain requiring a painkiller, whereas abdominal pain that necessitated a painkiller occurred in $39 \%(62 / 157)$ of the CG patients $(P<$ $0.05)$.

\section{Discussion}

Based on the results of this pilot study, local lidocaine injection into the sm layer was demonstrated to be safe during ESDs for EGC patients under sedation. The safety and efficacy of lidocaine as preemptive analgesia has already been assessed and proven in the surgical field, particularly with respect to laparoscopic surgery, and it is now commonly accepted that local anesthesia is effective during certain surgical procedures, and it is used accordingly [14-17]. Although lidocaine has generally been associated with a number of adverse reactions, such as respiratory depression, hypotension, convulsion, and arrhythmia, including cardiovascular collapse and bradycardia [18], there were no such complications observed in the present study. All the results related to complications, as well as the laboratory data, indicated that local lidocaine injection into the sm layer could be used safely during ESDs for EGCs performed under sedation.

ESD produces higher rates of en-bloc resections and tumor-free margins compared to conventional EMR. As a result, it has been proposed as the gold standard treatment for EGC, because it facilitates more accurate histological assessment and reduces the risk of tumor recurrence [19-21]. At the present time, the indications for ESD are in the process of being expanded; this will make it possible for even more EGC patients to be successfully treated without having to undergo open surgery.

ESD for large tumors is usually a prolonged procedure requiring higher doses of sedative and pain-control drugs such as midazolam and pentazocine, but there have been no published reports as yet addressing the problem of epigastric pain associated with ESDs. In our study, patient abdominal discomfort was considerably lower in the LG, most likely because of the immediate local anesthesic effect of lidocaine, as evidenced by the significantly lower mean total dose of pentazocine.

Preemptive analgesia is defined as preventing or reducing the memory of nociceptive stimuli in the central nervous system, utilizing analgesic methods performed prior to such nociceptive stimuli, with a resultant decrease in the need for postoperative analgesics. Recent research on postoperative pain control has led to the development of the concept of preemptive analgesia, in which pain management begins at the preoperative stage so as to decrease the severity of pain in the postoperative period, by applying analgesic methods 
before the onset of nociceptive stimuli [13, 22-24]. Based on this conceptual approach, local anesthesia can also have a preemptive analgesic effect, so it is likely that in the LG patients in our study the lidocaine injections had elevated their pain thresholds after completion of their ESDs. This, in turn, resulted in these patients not complaining of abdominal pain on the day of their procedures, and having fewer pain-related comments and milder pain on the day after the procedure.

The mean dose of midazolam required in the LG was lower than that in the CG, although the difference was not statistically significant, but the mean dose of pentazocine in the LG was significantly lower than that in the CG. This suggests that local lidocaine injection could reduce the amount of pentazocine required by locally controlling a patient's pain perception, thus resulting in less patient movement and fewer delays in the ESD caused by such movement. Fewer delays and less time spent administering sedative and pain-control drugs during a lengthier ESD procedure, combined with an actual reduction in the doses of such drugs, could reduce the risk of respiratory and other drug-related complications caused by oversedation.

In our study, none of the LG patients reported any abdominal pain on the day of their ESDs, indicating the probable effectiveness of local lidocaine injections for pain control during and immediately after ESD. Given lidocaine's characteristic feature of controlling pain for a only a short period, its local injection into the sm layer appears to be an effective method for pain management during and immediately following ESD, but further investigation of other longer-acting local analgesics is recommended.

Our investigation was a small pilot study of a lidocaine-treated group that was retrospectively compared to a considerably larger historical control group. A randomized control study will be necessary in the future to reliably assess the effectiveness of the particular technique that we have described. While the assessment and measurement of pain are very important considerations for both patients and physicians, pain tolerance varies greatly among patients, so further investigation will be required in accordance with the basic philosophy of preemptive analgesia.

In conclusion, local lidocaine injections into the sm layer during ESDs in EGC patients under sedation are safe. This study indicated that such lidocaine injections have a beneficial effect on local pain control during ESDs and in the immediate post-procedure period.

\section{References}

1. Oda I, Gotoda T, Hamanaka H, Eguchi T, Saito Y, Matsuda T, et al. Endoscopic submucosal dissection for early gastric cancer: technical feasibility, operation time and complications from a large consecutive series. Dig Endosc 2005;17:54-8.

2. Patel S, Vargo JJ, Khandwala F, Lopez R, Trolli P, Dumot JA, et al. Deep sedation occurs frequently during elective endoscopy with meperidine and midazolam. Am J Gastroenterol 2005;100: 2689-95.

3. Karaaslan D, Sivaci RG, Akbulut G, Dilek ON. Preemptive analgesia in laparoscopic cholecystectomy: a randomized controlled study. Pain Pract 2006;6:237-41.

4. Goldstein A, Grimault P, Henique A, Keller M, Fortin A, Darai E. Preventing postoperative pain by local anesthetic instillation after laparoscopic gynecologic surgery: a placebo-controlled comparison of bupivacaine and ropivacaine. Anesth Analg 2000;91: 403-7.

5. Einarsson JI, Sun J, Orav J, Young AE. Local analgesia in laparoscopy: a randomized trial. Obstet Gynecol 2004;104:1335-9.

6. Japanese Gastric Cancer Association. Japanese classification of gastric carcinoma - 2nd English edition. Gastric Cancer 1998;1: $10-24$.

7. Gotoda T, Yamamoto H, Soetikno RM. Endoscopic submucosal dissection of early gastric cancer. J Gastroenterol 2006;41:929_ 42.

8. Gotoda T, Kondo H, Ono H, Saito Y, Yamaguchi H, Saito D, et al. A new endoscopic mucosal resection (EMR) procedure using an insulation-tipped diathermic (IT) knife for rectal flat lesions. Gastrointest Endosc 1999;50:560-3.

9. Gotoda T. Endoscopic diagnosis and treatment for early gastric cancer. Cancer Review: Asia-Pacific 2004;2:17-37.

10. Ono H, Kondo H, Gotoda T, Shirao K, Yamaguchi H, Saito D, et al. Endoscopic mucosal resection for treatment of early gastric cancer. Gut 2001;48:225-9.

11. Polk JW, Bailey AH. Topical anesthesia for endoscopy; evaluation of prilocaine (Citanest), a new local anesthetic. Dis. Chest 1967;51:293-7.

12. Mather LE, Tucker GT, Murphy TM, Stanton-Hicks MD, Bonica JJ. The effects of adding adrenaline to etidocaine and lignocaine in extradural anaesthesia. II: Pharmacokinetics. Br J Anaesth 1976:48:989-94.

13. Garwood S, Reeder M, Mackenzie IZ, Guillebaud J. Tubal surface lidocaine mediates pre-emptive analgesia in awake laparoscopic sterilization: a prospective, randomized clinical trial. Am J Obstet Gynecol 2002;186:383-8.

14. Smith G. Intraperitoneal administration of analgesia: is this practice of any utility? Br J Anaesth 2002;89:535-7.

15. Elhakim M, Elkott M, Ali NM, Tahoun HM. Intraperitoneal lidocaine for postoperative pain after laparoscopy. Acta Anaesthesiol Scand 2000;44:280-4.

16. Carbonell AM, Harold KL, Mahmutovic AJ, Hassan R, Matthews $\mathrm{BD}$, Kercher $\mathrm{KW}$, et al. Local injection for the treatment of suture site pain after laparoscopic ventral hernia repair. Am Surg 2003;69:688-91.

17. Zullo F, Pellicano M, Cappiello F, Zupi E, Marconi D, Nappi C. Pain control after microlaparoscopy. J Am Assoc Gynecol Laparosc 1998;5:161-3.

18. Sweetman SC, Blake PS, McGlashan JM, Neathercoat GC, Parsons AV. Lidocaine. In: Martindale: the complete drug reference. 35th ed. London/Chicago: Pharmaceutical Press; 2006. p. 1702-6.

19. Rembacken BJ, Gotoda T, Fujii T, Axon ATR. Endoscopic mucosal resection. Endoscopy 2001;33:709-18.

20. Soetikno RM, Gotoda T, Nakanishi Y, Soehendra N. Endoscopic mucosal resection. Gastrointest Endosc 2003;57:567-79.

21. Eguchi T, Gotoda T, Oda I, Hamanaka H, Hasuike N, Saito D. Is endoscopic one-piece mucosal resection essential for early gastric cancer? Dig Endosc 2003;15:113-6.

22. Kedek A, Derbent A, Uyar M, Bilgen C, Uyar M, Kirazli, et al. Pre-emptive effect of ibuprofen syrup and lidocaine infiltration on post-operative analgesia in children undergoing adenotonsillectomy. J Int Med Res 2005;33:188-95. 
23. Homer JJ, Swallow J, Semple P. Audit of pain management at home following tonsillectomy in children. J Laryngol Otol 2001;115:205-8

24. Wu CT, Yett CC, Yu JC, Lee MMS, Tao PL, Ho ST, et al. Preincisional epidural ketamine, morphine and bupivacaine com- bined with epidural and general anaesthesia provides pre-emptive analgesia for upper abdominal surgery. Acta Anasthesiol Scand 2000;44:63-8. 